\title{
Needle tract recurrence of hepatocellular carcinoma after liver transplantation
}

Background-Percutaneous ultrasound guided needle aspiration biopsy is an efficient procedure for the diagnosis of hepatocellular carcinoma (HCC). However, this procedure may be responsible for metastatic seeding in the needle tract. ${ }^{1}$ We present a case of metastatic subcutaneous seeding after orthotopic liver transplantation (OLT) for cirrhosis complicated by HCC.

Case report - A 29 year old man with a past history of chronic hepatitis C and alcoholism was admitted in July 1987 for hepatocellular insufficiency. The diagnosis of cirrhosis was confirmed by liver biopsy. In February 1997, a $30 \mathrm{~mm}$ intrahepatic focal lesion was discovered at ultrasonographic examination in segment III. Serum alpha fetoprotein (AFP) level was normal. An ultrasound guided biopsy was performed using an 18 gauge needle. Histological examination showed HCC. In April 1997, OLT was performed. Examination of the liver explant showed a non-encapsulated, moderately differentiated HCC, measuring $3 \times 8 \times 12 \mathrm{~cm}$, associated with a large intrahepatic venous extension. Immunosuppressive treatment consisted of tacrolimus, methylprednisolone, and azathioprine. Adjuvant epiadriamycin chemotherapy was performed. In September 1999, 29 months after OLT, the patient presented with a large mass located in the subcutaneous fat at the site of the initial needle puncture. No intrahepatic lesion was detected. The subcutaneous nodule was resected. Histological examination confirmed the metastatic HCC. The lesion was $4.5 \mathrm{~cm}$ in diameter, ill limited, and invaded the adjacent striated muscle.

Discussion - The incidence of implantation metastases after fine needle diagnostic procedures has been estimated, according to multicentre questionnaires, to be less than $1 / 10000 .^{2}$ Several factors are suspected of increasing the incidence of needle tract recurrence: large needle diameter, high number of passes, and absence of surrounding normal liver parenchyma. ${ }^{2}$ Less than 30 needle tract seedings after biopsy of HCC have been reported during the past 16 years. ${ }^{1}$ However, the incidence is likely to be underestimated as most cases were probably not reported. Two recent comprehensive follow up studies from Japan and France have found needle tract seeding in $2 \%$ and $2.66 \%$ of patients, respectively. ${ }^{3}{ }^{4}$ Recurrent subcutaneous seeding of HCC after OLT has been very rarely reported. ${ }^{5}$ In our institution, between 1991 and 1999 OLT was performed in 44 patients presenting with HCC. Fine needle aspirates and/or biopsies were obtained in 31 of these patients. In our series, the

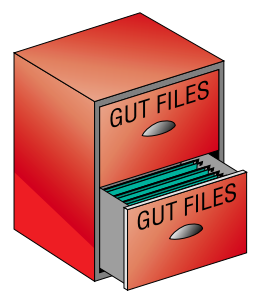
incidence of needle tract recurrence was therefore $3.22 \%$, which compares well with previously reported data.

The risk of needle tract seeding is of particular concern in patients presenting with small HCC for whom long survival may be expected after surgical resection or OLT. In such cases, a combination of clinical and imaging features, including the results of AFP monitoring, spiral computed tomography, or magnetic resonance imaging may be highly suggestive of the diagnosis, and may lead to re-evaluation of the role of needle biopsy.

Conclusion-Our report strongly suggests that the role of needle biopsy to confirm HCC in patients suitable for OLT must be thoroughly evaluated.

J DUMORTIER

C LOMBARD-BOHAS

P-J VALETTE

O BOILLOT

Fédération des Spécialités Digestives,

Hôpital Edouard Herriot, Lyon, France

J DUMORTIER

O BOILLOT

Unité de Transplantation Hépatique,

Hôpital Edouard Herriot, Lyon, France

J-Y SCOAZEC

F BERGER

Laboratoire Central d'Anatomie et Cytologie Pathologiques,

Hopital Edouard Herriot, Lyon, France

S CLAUDEL-BONVOISIN

Service de Gastroentérologie,

Centre Hospitalier Lyon Sud, Pierre Bénite, France

Correspondence to: Dr J Dumortier, INSERM U45, Pavillon H, Hôpital Edouard Herriot, 5 place d'Arsonval, 69437 Lyon cedex 03, France.

Email: jdumortier@1yon151.inserm.fr

1 Schotman SN, De Man RA, Stoker J, Zondervan PE, Ijzermans JN. Subcutaneous seeding of hepatocellular carcinoma after percutaneous needle biopsy. Gut 1999;45:626-7.

Smith EH. Complications of percutaneous abdominal fine-needle biopsy. Review. Radiology 1991;178:253-8.

3 Huang GT, Sheu JC, Yang PM, et al. Ultrasound-guided cutting biopsy for the diagnosis of hepatocellular carcinoma - a study based on 420 patients. f Hepatol 1996;25:334-8.

4 Chapoutot C, Perney P, Fabre D, et al. Essaimages tumoraux sur le trajet de ponctions écho-guidées de carcinomes hépatocellulaires. Etude de 150 malades. Gastroenterol Clin Biol 1999;23:552-6.

5 McPeake JR, O'Grady JG, Zaman S, et al. Liver transplantation for primary hepatocellular carcinoma: tumor size and number determine outcome. $\mathcal{F}$ Hepatol 1993;18:226-34. 